\title{
Immunological features of patients affected by Barraquer-Simons syndrome
}

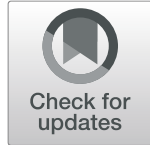

Fernando Corvillo ${ }^{1,2^{*}}$ D, Giovanni Ceccarini ${ }^{3}$, Pilar Nozal ${ }^{1,2,4}$, Silvia Magno ${ }^{3}$, Caterina Pelosini ${ }^{3}$, Sofía Garrido $^{1,2,4}$, Alberto López-Lera ${ }^{1,2}$, Manuela Moraru ${ }^{5}$, Carlos Vilches ${ }^{5}$, Silvia Fornaciari ${ }^{6}$, Sabrina Gabbriellini ${ }^{6}$, Ferruccio Santini ${ }^{3}$, David Araújo-Vilar ${ }^{7}$ and Margarita López-Trascasa ${ }^{1,8}$

\begin{abstract}
Background: C3 hypocomplementemia and the presence of C3 nephritic factor (C3NeF), an autoantibody causing complement system over-activation, are common features among most patients affected by Barraquer-Simons syndrome (BSS), an acquired form of partial lipodystrophy. Moreover, BSS is frequently associated with autoimmune diseases. However, the relationship between complement system dysregulation and BSS remains to be fully elucidated. The aim of this study was to provide a comprehensive immunological analysis of the complement system status, autoantibody signatures and HLA profile in BSS. Thirteen subjects with BSS were recruited for the study. The circulating levels of complement components, C3, C4, Factor B (FB) and Properdin (P), as well as an extended autoantibody profile including autoantibodies targeting complement components and regulators were assessed in serum. Additionally, HLA genotyping was carried out using DNA extracted from peripheral blood mononuclear cells.
\end{abstract}

Results: C3, C4 and FB levels were significantly reduced in patients with BSS as compared with healthy subjects. C3NeF was the most frequently found autoantibody (69.2\% of cases), followed by anti-C3 (38.5\%), and anti-P and antiFB (30.8\% each). Clinical data showed high prevalence of autoimmune diseases (38.5\%), the majority of patients (61.5\%) being positive for at least one of the autoantibodies tested. The HLA allele DRB1*11 was present in 54\% of BSS patients, and the majority of them (31\%) were positive for *11:03 (vs 1.3\% allelic frequency in the general population).

Conclusions: Our results confirmed the association between BSS, autoimmunity and C3 hypocomplementemia. Moreover, the finding of autoantibodies targeting complement system proteins points to complement dysregulation as a central pathological event in the development of BSS.

Keywords: Complement system, Lipodystrophy, Barraquer-Simons syndrome, C3 nephritic factor, Autoimmunity, Acquired partial lipodystrophy

\section{Background}

Lipodystrophies are a heterogeneous group of rare diseases characterized by loss of adipose tissue. They can be divided into generalized, partial or localized depending on the extent of fat loss. Additionally, both, generalized and partial forms can be classified as inherited and acquired [1]. Barraquer-Simons syndrome (BSS)

\footnotetext{
*Correspondence: fcorvillo@yahoo.es

${ }^{1}$ Complement Research Group, Hospital La Paz Institute for Health Research (IdiPAZ), La Paz University Hospital, Paseo de la Castellana, 261, 28046 Madrid, Spain

${ }^{2}$ Center for Biomedical Network Research on Rare Diseases (CIBERER U754), Madrid, Spain

Full list of author information is available at the end of the article
}

(ORPHA: 79087), is an acquired form of partial lipodystrophy, characterized by bilateral symmetrical loss of adipose tissue that begins in the face and may variably spreads to neck, shoulders, arms and trunk, keeping intact the adipose tissue of the lower extremities [2]. Sometimes, after puberty, mainly in women, adipose tissue is hypertrophic in the lower extremities, causing a regional disproportion. Females are more affected than males with a ratio of $4: 1[3,4]$. There is usually no family history of lipodystrophy. Onset of the disease usually occurs during childhood or adolescence, sometimes after viral infections [5]. As opposed to other types of lipodystrophies, metabolic diseases and associated 
comorbidities appear less common in patients with BSS $[4,5]$, though some of them can present severe metabolic complications [6]. Several reports have shown the association of BSS with autoimmune diseases in a minority of patients, in particular systemic lupus erythematosus and dermatomyositis. Other autoimmune diseases that are less frequently associated with BSS include autoimmune thyroiditis, localized scleroderma, idiopathic thrombocytopenic purpura and Sjögren's syndrome, among others $[4,5,7]$.

A common feature among patients with BSS is the C3 hypocomplementemia [5]. Sissons et al. [8] provided a comprehensive study of the association of BSS with complement dysregulation. In that study, most patients with C3 consumption had detectable levels of an IgG/IgM autoantibody called $\mathrm{C} 3$ nephritic factor $(\mathrm{C} 3 \mathrm{NeF})$. C3NeF stabilizes the enzymatic complex $\mathrm{C} 3$ convertase $(\mathrm{C} 3 \mathrm{bBb})$, which causes unopposed activation of the alternative pathway (AP) of the complement system [9]. The presence of this autoantibody has been associated with a rare entity named C3 glomerulopathy (C3G) [10]. C3G is a kidney disease characterized by $\mathrm{C} 3$ predominant staining and minimal or absent immunoglobulin staining observed on renal biopsy by immunofluorescence [11]. The incidence of C3G is approximately 1 per million per year [12]. C3G is divided into dense deposit disease (DDD) and C3 glomerulonephritis (C3GN), based on electron microscopy findings [13]. The most important adverse outcome associated with the diagnosis of C3G is progression to end-stage renal disease, which occurs within 10 years from diagnosis in $\sim 70 \%$ of affected children and $30-50 \%$ of affected adults [13]. Concerning patients with BSS, 20\% of the them eventually develops C3G [2, 4].

Adipocytes are the main source of synthesis and secretion of a serine protease called adipsin or factor D (FD) [14-16]. This enzyme is considered the key limiting factor for the activation of the AP. FD cleaves factor B (FB) when it forms part of the $\mathrm{C} 3$ pro-convertase $(\mathrm{C} 3 \mathrm{bB})$, generating the active AP C3 convertase. Moreover, adipocytes also express other components of the AP, such as $\mathrm{C} 3, \mathrm{FB}$ and complement regulators like properdin $(\mathrm{P})$, factor $\mathrm{H}(\mathrm{FH})$ and factor I (FI) [17-20]. Furthermore, local complement activation appears to be involved in the synthesis of triglycerides and adipocyte differentiation [17]. Although the connection between complement abnormalities and renal disease has been established, the exact mechanism of fat loss remains unclear. Mathieson and collaborators demonstrated that $\mathrm{C} 3 \mathrm{NeF}$ could induce complement-mediated lysis of adipocytes in vitro [21]. However, the fact that only a small proportion of patients with $\mathrm{C} 3 \mathrm{NeF}$ develop lipodystrophy remains unexplained.

We herein describe the immunological and clinical characteristics of a group of 13 patients diagnosed with
BSS. Our results show that C3 hypocomplementemia and $\mathrm{C} 3 \mathrm{NeF}$ autoantibodies are present in approximately $70 \%$ of the patients; besides, we found the presence of other autoantibodies against individual complement components. Immunological studies were extended with Human Leukocyte antigen (HLA) phenotyping and the screening of autoimmune markers.

\section{Results \\ Demographic and clinical data}

Demographic details and basic clinical information are listed in Table 1. In our cohort, patients were mainly female (ratio 5.5:1) and the mean age at the time of the study was 33 (range 8-76). Lipodystrophy onset occurred during childhood (mean 8 years) in most cases and only one patient, BSS6, developed the disease in adulthood (41 years old) after the implantation of orthodontic brackets. Most of the patients conserved the body mass index (BMI) into normal range, and some them registered slight decreases of the percentage of whole total fat mass. Regarding glucidic metabolism, one patient had elevated insulin and HbA1c levels and was diagnosed with diabetes. Mild low leptin levels (8.03 ng/ $\mathrm{ml}$; normal range: $15.3 \pm 8.1$ standard deviation) were present in one patient and triglycerides, total cholesterol and LDL-cholesterol levels were elevated in two patients. Three patients were diagnosed with fatty liver, linked to higher concentrations of AST and ALT transaminases. BSS1 suffered a hepatitis B virus (HBV) infection that lead to the deterioration of liver function with elevation of transaminases and development of hepatocellular carcinoma which shortly ended in death. Concerning cardiovascular diseases, three patients developed arterial hypertension. Finally, BSS1, BSS3 and BSS9 were diagnosed with DDD, IgA nephropathy and C3GN, respectively.

\section{Complement system profile in patients with BSS}

C3 levels were significantly reduced $(P=0.01)$ in the group of patients with BSS [median $31.7 \mathrm{mg} / \mathrm{dl}$ (IQR, 10.4$100.8)]$ in comparison with healthy donors (NHS) [100.5 $\mathrm{mg} / \mathrm{dl}$ (85.2-111.5)] (Fig. 1a). The same applies for C4 [BSS, $16.5 \mathrm{mg} / \mathrm{dl}$ (14.1-23.5); NHS, $25 \mathrm{mg} / \mathrm{dl}$ (19.3-27.7); $P=0.04$ ] and FB levels [BSS, $16.5 \mathrm{mg} / \mathrm{dl}$ (14.1-23.5); NHS, $25 \mathrm{mg} / \mathrm{dl}$ (19.3-27.7); $P=0.04$ ] (Fig. 1b), that presented significant differences between both groups.

Conversely, similar P levels were found in controls and BSS patients [BSS, $26.7 \mathrm{mg} / \mathrm{dl}(23.9-29.7)$ versus NHS, $28.7 \mathrm{mg} / \mathrm{dl}$ (24.9-32.3), $P=0.22$ ] (Fig. 1d).

\section{C3NeF is not the only autoantibody against AP components in patients with BSS}

The frequencies of these autoantibodies are shown in Table 2. C3NeF was the most frequent autoantibody 
Table 1 Clinical and demographical data of patients with BSS

\begin{tabular}{|c|c|c|c|c|c|c|c|c|c|c|c|c|c|c|}
\hline Variable & BSS1 & BSS2 & BSS3 & BSS4 & BSS5 & BSS6 & BSS7 & BSS8 & BSS9 & BSS10 & BSS11 & BSS12 & BSS13 & Reference \\
\hline Sex & $\mathrm{F}$ & $\mathrm{F}$ & $\mathrm{F}$ & $F$ & $F$ & $F$ & $F$ & $F$ & M & M & $F$ & $\mathrm{~F}$ & $F$ & \\
\hline Age (years) & 76 & 13 & 15 & 8 & 49 & 42 & 41 & 44 & 52 & 11 & 62 & 10 & 12 & \\
\hline $\begin{array}{l}\text { Lipodystrophy onset } \\
\text { (years) }\end{array}$ & N/A & 2 & 5 & 4 & 9 & 41 & 7 & 3 & 6 & 7 & 8 & 7 & 7 & \\
\hline BMI (kg/m2) & 24.3 & 19.3 & 18 & 16.3 & 19.8 & 22.3 & 33.8 & 22.7 & 23.5 & 21.3 & 26.1 & 16.5 & 22.7 & $18-25$ \\
\hline$\%$ total fat ${ }^{a}$ & N/A & N/A & 17.7 & 22 & 20.3 & 27.8 & 41.8 & 23 & 28.7 & 33.2 & 29.1 & 31.2 & 42.6 & $\begin{array}{l}25-31 \\
\text { (Women) } \\
18-24 \\
\text { (Men) }\end{array}$ \\
\hline Acanthosis & No & No & No & No & No & No & No & No & No & No & No & No & No & Yes/No \\
\hline Glucose (mg/dL) & 79 & 85 & 69 & 86 & 83 & 93 & 82 & 102 & 84 & 90 & 107 & 94 & 84 & $60-110$ \\
\hline Insulin $(\mu \mathrm{U} / \mathrm{mL})$ & 15.8 & 10.4 & 10.7 & 13.5 & N/A & N/A & 18.5 & 9.8 & 13 & 14.3 & 33.3 & 9 & 4.74 & $2.6-24.9$ \\
\hline Diabetes & No & No & No & No & No & No & No & No & No & No & Yes & No & No & Yes/No \\
\hline $\mathrm{HbA1c}(\%)$ & N/A & 4.8 & N/A & 5.3 & 5.2 & N/A & 5.4 & N/A & N/A & 5.2 & 6.4 & 5.1 & 5.2 & $4.0-5.5$ \\
\hline Hypoleptinemia & No & No & Yes & No & No & No & No & No & No & No & No & No & No & Yes/No \\
\hline Triglycerides (mg/dL) & 106 & 79 & 110 & 89 & 78 & 66 & 105 & 210 & 445 & 61 & 265 & 79 & 53 & $25-115$ \\
\hline Total colesterol (mg/dL) & 129 & 145 & 122 & 163 & 238 & 183 & 158 & 120 & 245 & 112 & 175 & 167 & 159 & $110-230$ \\
\hline HDL-cholesterol (mg/dL) & 57 & 62 & N/A & 42 & 69 & 70 & 63 & 35 & 45 & 45 & 37 & 44 & 66 & $40-70$ \\
\hline LDL-cholesterol (mg/dL) & 120 & 109 & N/A & 112 & 153.4 & 100 & 74 & 111 & 163 & 64 & 110 & 126 & 101 & $50-129$ \\
\hline Fatty liver ${ }^{\mathrm{b}}$ & No & No & No & No & No & No & Yes & No & No & Yes & Yes & No & No & Yes/No \\
\hline ALT (UI/L) & 33 & 28 & 13 & 24 & 31 & 20 & 60 & 21 & 21 & 15 & 24 & 22 & 28 & $5-31$ \\
\hline AST (UI/L) & 51 & 24 & 23 & 33 & 26 & 19 & 39 & 15 & 15 & 24 & 25 & 25 & 20 & $5-32$ \\
\hline Hypertension & Yes & No & No & No & No & No & No & No & Yes & No & Yes & No & No & Yes/No \\
\hline Renal disease & Yes (DDD) & No & Yes (IgAN) & No & No & No & No & No & Yes (C3GN) & No & No & No & No & Yes/No \\
\hline
\end{tabular}

Abbreviations: $F$ female, $M$ male, BMI Body Mass Index, HbA1c Glycohemoglobin, ALT Alanine aminotransferase, AST Aspartate aminotransferase; DDD Dense deposit disease, IgAN IgA nephropathy, C3GN C3 glomerulonephritis, N/A Not available

a Percentage of total fat was measured using dual-energy $x$-ray absorptiometry

${ }^{\mathrm{b}}$ Liver steatosis was assessed by means of ultrasonography

(69.2\% of cases) observed in sera from patients with BSS, in accordance with previous observations [2, 4, 5]. Additional autoantibodies against the AP C3 convertase (C3bBbP) components were also detected. Autoantibodies against $\mathrm{P}$ were the most prevalent $(38.5 \%$ of cases), followed by anti-C3 and anti-FB autoantibodies (30.8\% for both). Moreover, the presence of one or more of these autoantibodies was always associated with C3 consumption. However, none of them showed significant reactivity against complement negative regulators $\mathrm{FH}$ and FI. Autoantibodies against complement proteins were not found in the 20 healthy subjects analyzed as control population. A schematic representation of these autoantibodies and their antigens is shown in Additional file 1: Figure S1.

\section{Profile of HLA alleles in patients with BSS}

To investigate the immunogenetic variables potentially associated with BSS we determined the HLA class I and class II phenotypes (Table 3 ). The very low sample size and the enormous polymorphism of the HLA system preclude a proper, statistically powered, study of association. However, examination of phenotypes reveals that: (i) no HLA allele was shared by all, or a vast majority of BSS patients; (ii) notwithstanding, 3/9 Spanish and 1/4 Italian patients (ca. 31\%) carried allele DRB1*11:03, reported in Spaniards at an allelic frequency of $\sim 1.3 \%$ (calculated carrier frequency $\sim 2.6 \%$ [22]), the global frequency of all DRB1*11 alleles in BSS patients being 54\%; and (iii), no patient carried B*07, B*14 and DRB1*15 alleles, all common in European Caucasoids. Future studies in larger patients' cohorts should address whether these observations reflect a real predisposing effect.

\section{Autoimmunity and autoimmune diseases are prevalent in patients with BSS}

The profile of autoantibodies and related diseases are summarized in Table 4. Clinical data from the 13 patients showed a high prevalence of autoimmune diseases (38.5\%) including Hashimoto's thyroiditis $(n=2)$, vitiligo $(n=1), \quad$ Sjögren's syndrome $(n=1)$ and rheumatoid 
A

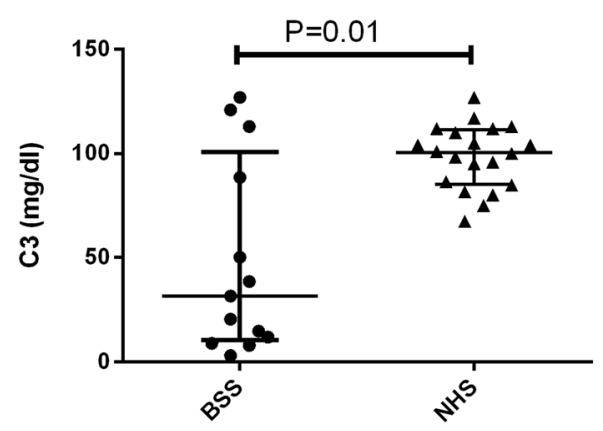

C

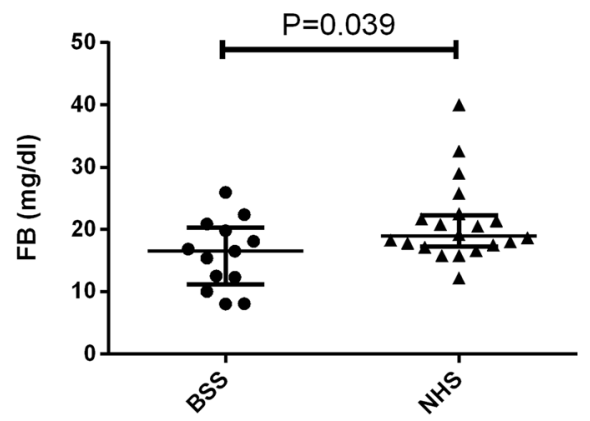

B

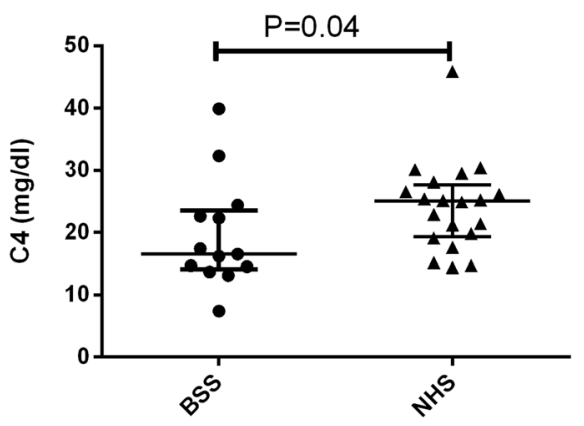

D

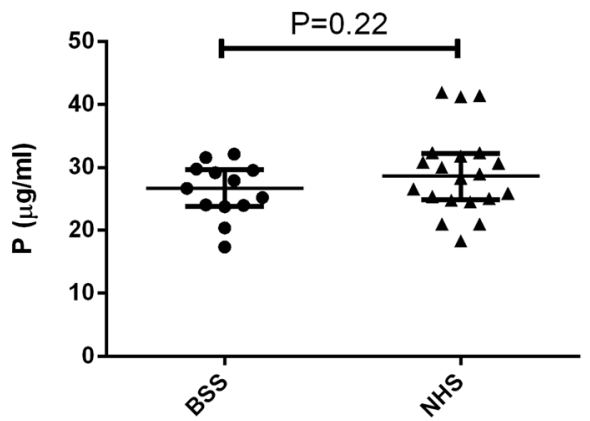

Fig. 1 Complement profile in Barraquer-Simons syndrome (BSS) cohort. (a) C3 levels, (b) C4 levels (c) Factor B (FB) levels and (d) properdin (P) levels. Data are represented by median with interquartile ranges. The results of the BSS cohort $(n=13)$ are compared with a group of 20 healthy subjects (NHS). Data is statistically significant for $P<0.05$

Table 2 Frequencies of autoantibodies against complement proteins in patients affected by BSS

\begin{tabular}{|c|c|c|c|c|c|c|c|}
\hline & $\mathrm{C} 3 \mathrm{NeF}$ & anti-C3 & anti-FB & anti-P & anti-FI & anti-FH & C3 (mg/dl) \\
\hline BSS1 & Positive & Positive & Negative & Negative & Negative & Negative & 3.0 \\
\hline BSS2 & Positive & Positive & Negative & Negative & Negative & Negative & 11.9 \\
\hline BSS3 & Positive & Positive & Positive & Positive & Negative & Negative & 31.7 \\
\hline BSS4 & Positive & Positive & Negative & Negative & Negative & Negative & 7.9 \\
\hline BSS5 & Positive & Negative & Negative & Negative & Negative & Negative & 14.8 \\
\hline BSS6 & Negative & Negative & Negative & Negative & Negative & Negative & 88.5 \\
\hline BSS7 & Positive & Negative & Positive & Positive & Negative & Negative & 38.7 \\
\hline BSS8 & Negative & Negative & Negative & Negative & Negative & Negative & 121.0 \\
\hline BSS9 & Positive & Negative & Positive & Positive & Negative & Negative & 20.6 \\
\hline BSS10 & Positive & Negative & Positive & Positive & Negative & Negative & 50.2 \\
\hline BSS11 & Negative & Negative & Negative & Negative & Negative & Negative & 127.0 \\
\hline BSS12 & Positive & Negative & Negative & Positive & Negative & Negative & 8.9 \\
\hline \multirow[t]{2}{*}{ BSS13 } & Negative & Negative & Negative & Negative & Negative & Negative & 113.0 \\
\hline & 9/13 (69.2\%) & $4 / 13(30.8 \%)$ & 4/13 (30.8\%) & $5 / 13(38.5 \%)$ & $0 / 13(0 \%)$ & 0/13 (0\%) & Range: $75-135 \mathrm{mg} / \mathrm{d}$ \\
\hline
\end{tabular}


Table 3 HLA profiles of patients affected by BSS

\begin{tabular}{|c|c|c|c|c|c|c|c|}
\hline \multicolumn{8}{|l|}{ Patient } \\
\hline BSS1 & $A^{*} 01: 01, * 30: 02$ & $B^{*} 08: 01, * 18: 01$ & $C^{*} 05: 01, * 07: 01$ & DRB $1 * 03: 01$ & $\mathrm{DQB1}{ }^{* 02: 01}$ & DQA $1 * 05: 01$ & $\mathrm{DPB} 1 * 01: 01, * 02: 02$ \\
\hline BSS2 & $A^{*} 02: 01,{ }^{*} 11: 01$ & $\begin{array}{l}B^{*} 35: 01, * 57: 03 / \\
B^{*} 35: 04, * 57: 01\end{array}$ & $C^{*} 04: 01,{ }^{*} 07: 01$ & DRB1*11:03,*14:54 & DQB1 ${ }^{*} 03: 01,{ }^{*} 05: 03$ & DQA $1 * 01: 04,{ }^{*} 05: 05 / 09$ & $\mathrm{DPB} 1 * 04: 01$ \\
\hline BSS3 & $A^{*} 03: 01,{ }^{*} 11: 01$ & $B^{*} 27: 05, * 35: 01$ & $C^{*} 01: 02,{ }^{*} 04: 01$ & DRB $1 * 01: 01, * 04: 03$ & DQB1 ${ }^{*} 03: 02,{ }^{*} 05: 01$ & DQA $1 * 01: 01, * 03: 01$ & $\mathrm{DPB} 1 * 04: 01$ \\
\hline BSS4 & $A^{*} 02: 01,{ }^{*} 24: 02$ & $B^{*} 40: 06, * 49: 01$ & $C^{*} 07: 01, * 15: 02$ & DRB $1 * 01: 01, * 11: 03$ & DQB1 ${ }^{*} 03: 01,{ }^{*} 05: 01$ & DQA $1 * 01: 01,{ }^{*} 05: 05 / 09$ & $\mathrm{DPB} 1 * 02: 01, * 04: 01$ \\
\hline BSS5 & $A^{*} 03: 01,{ }^{*} 29: 02$ & $B^{*} 18: 01, * 44: 02$ & $C^{*} 05: 01$ & DRB $1 * 03: 01, * 11: 01$ & DQB1 ${ }^{*} 02: 01,{ }^{*} 03: 01$ & DQA $1 * 05: 01,{ }^{*} 05: 05 / 09$ & $\mathrm{DPB} 1 * 04: 01$ \\
\hline SS6 & $A^{*} 11: 01, * 24: 02$ & $B^{*} 53: 01, * 57: 01$ & $C^{*} 04: 0$ & DRB $1 * 07: 01, * 13: 02$ & DQB1 ${ }^{*} 03: 03,{ }^{*} 06: 04$ & DQA $1 * 01: 02, * 02: 01$ & $\mathrm{DPB} 1{ }^{*} 03: 01,{ }^{*} 04$ \\
\hline BSS7 & $A^{*} 29: 02,{ }^{*} 68: 01$ & $B^{*} 44: 02, * 49: 01$ & $C^{*} 07: 01, * 07: 04$ & DRB1 ${ }^{*} 04: 01, * 07: 01$ & DQB1 ${ }^{*} 02: 02,{ }^{*} 03: 01$ & DQA $1 * 02: 01, * 03: 01$ & $\mathrm{DPB} 1 * 04: 01$ \\
\hline BSS8 & $A^{*} 02: 01, * 24: 02$ & $B^{*} 15: 01, * 41: 02$ & $\begin{array}{l}C * 03: 03,{ }^{*} 17: 01 / \\
17: 03\end{array}$ & DRB1*11:03,*13:03 & $\mathrm{DQB1}{ }^{*} 03: 01$ & $\mathrm{DQA} 1 * 05: 05 / 09$ & $\mathrm{DPB} 1{ }^{*} 04: 01,{ }^{*} 04$ \\
\hline BSS9 & $A^{*} 32: 01,{ }^{*} 68: 01$ & $B^{*} 35: 08, * 51: 01$ & $C^{*} 04: 01, * 15: 02$ & DRB1*04:03,*13:01 & DQB1 ${ }^{*} 03: 02,{ }^{*} 06: 03$ & DQA $1 * 01: 03,{ }^{*} 03: 01$ & $\mathrm{DPB} 1{ }^{*} 02: 01,{ }^{*} 04:$ \\
\hline$S S 10$ & $A^{*} 02: 01, * 24: 02$ & $B^{*} 15: 01,{ }^{*} 18: 01$ & $C^{*} 03: 03, * 12: 03$ & DRB $1 * 04: 01, * 11: 04$ & DQB1 ${ }^{*} 03: 01,{ }^{*} 03: 02$ & DQA $1 * 03: 01,{ }^{*} 05: 05 / 09$ & n.s. \\
\hline BSS11 & $A^{*} 02: 01,{ }^{*} 24: 02$ & $B^{*} 35: 01, * 45: 01$ & $C^{*} 04: 01,{ }^{*} 07: 02$ & DRB1*11:01, ${ }^{*} 11: 03$ & DQB1*03:01 & DQA $1 * 05: 05 / 09$ & n.s. \\
\hline$S S 12$ & $A^{*} 02: 01$ & $B^{*} 27: 05, * 39: 06$ & $C^{*} 02: 02, * 07: 02$ & DRB1*08:01,*13:01 & DQB1 ${ }^{*} 04: 02,{ }^{*} 06: 03$ & DQA 1*01:03,*04:01 & n.s. \\
\hline BSS13 & $A^{*} 02: 01,{ }^{*} 11: 01$ & $B * 44: 02, * 55: 01$ & $C^{*} 03: 03,05: 01$ & DRB1*10:01, ${ }^{*} 16: 01$ & DQB1 ${ }^{*} 05: 01,{ }^{*} 05: 02$ & DQA $1 * 01: 01 / 04 / 05,{ }^{*} 01: 02$ & n.s. \\
\hline
\end{tabular}

The most likely HLA alleles at four-digit resolution, assigned as indicated in Materials and Methods, are displayed for each patient. Slashes represent ambiguities in the phenotype; n.s. not studied

arthritis $(n=1)$. Moreover, $61.5 \%$ of patients were positive for one or more autoantibodies tested and ANA were detected in $30.8 \%$ at a $1 / 160$ titer in all cases.

Two patients (BSS5 and BSS7) who were diagnosed with Hashimoto's thyroiditis were positive for anti- TG$\mathrm{Ab}$ and one of them (BSS7) for TPO-Ab. Patients BSS10 and BSS12 were positive for DAT or Coombs test but they were not affected with autoimmune hemolytic

Table 4 Clinical and laboratory markers of autoimmunity in patients affected by BSS

\begin{tabular}{lll}
\hline & Autoimmune markers & Autoimmune disease \\
\hline BSS1 & Anti-dsDNA indeterminated, RF & Rheumatoid arthritis \\
BSS2 & - & - \\
BSS3 & - & - \\
BSS4 & - & - \\
BSS5 & TG-Ab & Hashimoto's thyroiditis \\
BSS6 & - & - \\
BSS7 & TG-Ab, TPO-Ab & Hashimoto's thyroiditis, \\
& & vitiligo, Sjögren's syndrome \\
BSS8 & - & - \\
BSS9 & ANA (1:160) & - \\
BSS10 & DAT+ \\
BSS11 & ANA (1:160) & - \\
BSS12 & DAT+, ENA (Anti Ro52Ab), & - \\
BS513 & ANA (1:160) & - \\
APCA, ANA (1:160), GAD-Ab & -
\end{tabular}

Abbreviations: Anti-dsDNA anti-double stranded DNA, RF rheumatoid factor, $T G-A b$ thyroglobulin antibody, TPO-Ab thyroid peroxidase antibody, ANA antinuclear antibody, DAT Direct Antiglobulin Test, APCA anti-parietal cell antibody, GAD-Ab Glutamic Acid Decarboxylase autoantibodies; -, absent anemia at the time the study was performed. Another patient with Hashimoto's thyroiditis also presented with vitiligo and Sjögren's syndrome (BSS7). One patient had active rheumatoid arthritis with elevated rheumatoid factor (BSS1). APCA and Glutamic Acid Decarboxylase GAD-Ab were found in one patient (BSS13) but no related diseases have been developed at present. No dermatomyositis-related autoantibodies were detected in any patient.

\section{Discussion}

Abnormalities in the AP of the complement system are highly frequent in patients with BSS $[2,3,5,8]$. Presumably as a consequence of AP dysregulation, C3 hypocomplementemia has been reported in $70-80 \%$ of patients with BSS [3]. Moreover, this feature is widely established as a critical marker for the differential diagnosis of this type of lipodystrophy [4].

In the present study and in line with previous reports, C3 hypocomplementemia was found in $69.3 \%$ of the patients all of whom were positive for $\mathrm{C} 3 \mathrm{NeF}$ (Fig. 1a) $[5,8]$. Furthermore, BSS patients in our series also had significantly low FB levels as compared to controls (Fig. 1c). FB is a complement protein subjected to proteolytic cleavage by FD during the activation of the AP. FB levels may indeed be reduced in patients with $\mathrm{C} 3 \mathrm{NeF}$ but this is not a constant finding. This issue has been debated in several works but there are heterogeneous results concerning FB levels in the published series [23-25]. During $\mathrm{AP}$ activation, in the absence of $\mathrm{C} 3 \mathrm{NeF}, \mathrm{C} 3$ and $\mathrm{FB}$ consumption are usually correlated; however, $\mathrm{C} 3 \mathrm{NeF}$ breaks this correlation. One molecule of FB is needed for the 
assembly of one AP C3 convertase. C3NeF stabilizes the C3 convertase so that thousands of $\mathrm{C} 3$ molecules are proteolyzed by this complex leading to $\mathrm{C} 3$ consumption with no need for further convertase formation and subsequent FB consumption. This stabilizing effect of $\mathrm{C} 3 \mathrm{NeF}$ is heterogeneous among individuals, so it may be hypothesized that the decrease of FB levels is correlated with the stabilizing ability of the heterogeneous $\mathrm{C} 3 \mathrm{NeF}$ pool in each patient. Strikingly, P levels remained normal in our cohort (Fig. 1d). One possible explanation is that $\mathrm{P}$ is not the target of proteolytic cleavages and its consumption during the activation of the AP is unlikely.

Activation of the classic pathway (CP), resulting in low $\mathrm{C} 4$, has been described in some patients with acquired generalized lipodystrophy (AGL), though not being considered an specific feature of this pathology [26, 27]. In our study, C4 levels were significantly lower in BSS patients compared to controls (Fig. 1b). This observation suggests that, in BSS, as previously described, the activation of both CP and AP may occur [8]. The activation of $\mathrm{CP}$ could be explained as a consequence of the formation of antigen-antibody complexes. Interestingly, in patients who tested positive for the presence of antibodies against C3 (BSS1 to BSS4, see Table 2), one of the most abundant plasmatic proteins, lower $\mathrm{C} 4$ levels were found, probably as a consequence of the higher titers of circulating immune-complexes. However, further studies are needed to confirm this association.

In this report, age- and sex-matched healthy subjects were not available as controls, which is a limitation of the study. Other authors have previously shown that complement protein levels modify during aging; however, C3, C4, FB or P (proteins quantified in this work) do not exhibit significant variation with aging [28] and linear regression studies performed on our data have confirmed that aging is not influencing the results of the study (Additional file 1: Figure S2). Moreover, previous reports have established the normal variation range of C3 and C4 complement levels with age [29], and data presented here for patients with BSS are out of normal ranges, again indicating that the observed variation is not due to aging.

Females were overrepresented in both our patient and control cohorts (85 and 60\%, respectively). According to Gaya da Costa et al. [28], the levels of C3 and P are also significantly lower in women in comparison with men, which would result in significant bias in quantitative studies. To discard such effect, we compared the levels of $\mathrm{C} 3, \mathrm{C} 4, \mathrm{FB}$, and $\mathrm{P}$ between sexes, and no significant differences for any complement component were found in our cohort of healthy subjects (Additional file 1: Figure S3).

Our results demonstrate an association between the presence of autoantibodies directed against proteins of the AP and altered complement levels in patients with BSS. All of those patients with BSS and severe C3 consumption were positive for at least one of the analyzed autoantibodies the more prevalent being $\mathrm{C} 3 \mathrm{NeF}$ (Table 2). $\mathrm{C} 3 \mathrm{NeF}$ is associated with a predisposition to develop kidney disease in the medium to long term, which may explain why some patients in our series have developed C3G (BSS1 and BSS9) and IgA nephropathy (BSS3) (Table 1). C3G and IgAN are two diseases with different pathophysiological bases that share marked dysregulation of the complement system. Although associations between IgAN and glomerular and circulating markers of complement activation are established, the mechanism of complement activation and contribution to glomerular inflammation and injury is not defined. All reports had described that C3G is the main cause of renal disease in patients with BSS and in these cases activation of the AP is the main mechanism involved in complement dysregulation. In the IgAN, lectin and AP could be involved in the activation of the complement system. This case with IgAN and BSS could be the result of an association of two entities in which complement consumption should be mostly related with the presence of an IgG with $\mathrm{C} 3 \mathrm{NeF}$ activity. However, we cannot rule out this to be an accidental finding not related to the lipodystrophic phenotype.

Regarding the antibodies against additional complement proteins ( $\mathrm{C} 3, \mathrm{FB}$ and $\mathrm{P})$ it should be noted that they were detected only in patients who were also positive for $\mathrm{C} 3 \mathrm{NeF}$ (Table 2). Accordingly, it is reasonable to speculate that whatever the mechanism leading to the break of immunological tolerance, if the $\mathrm{C} 3 \mathrm{bBb}$ convertase (with or without $\mathrm{P}$ ) bears the neoepitope targeted by $\mathrm{C} 3 \mathrm{NeF}$, additional autoantibodies directed against single proteins of this complex could be concurrently generated. Of note, autoantibodies against negative regulators of the $\mathrm{AP}$ (FH and $\mathrm{FI}$ ) were not found in these patients (Table 2). Unlike C3NeF, whose importance in lipodystrophy has been largely studied and validated, autoantibodies against individual components of the C3 convertase have never been previously described in BSS. Anti-FB autoantibodies were first reported in a patient with DDD by Strobel and coleagues [30]. Their study revealed that they prevented the spontaneous dissociation of the $\mathrm{C} 3$ convertase of the AP and increased its normal half-life, thus causing systemic complement activation in the patient [30]. Additional patients with anti-FB and anti-C3b autoantibodies have since been reported in C3G and Ig-Associated Membranoproliferative Glomerulonephritis cohorts supporting their pathological role in the dysregulation of the AP [31, 32]. Therefore, further studies are needed in order to clarify if anti-FB and anti-C3b autoantibodies have a primary mechanistic role in the 
pathology of the lipodystrophy or if they rather secondarily arise as a consequence of the increase in circulating complement proteins produced due to unabated complement activation in the presence of C3NeF. A plausible possibility in line with the presence of $\mathrm{C} 3 \mathrm{NeF}$ and low C3 levels in our BSS cohort is that these autoantibodies synergistically promote further C3 convertase stabilization and C3 consumption in serum, similarly to what has already been described by Vasilev and colleagues for anti-C3 and anti-C3b autoantibodies in patients with lupus nephritis [33, 34].

Although the etiology of BSS is generally unknown, several studies have described its association with signs of autoimmunity $[5,7,35]$, a feature which is considered a supportive clinical criterion for the diagnosis to BSS [5]. Patients have been recruited in Italy and Spain, and autoantibody screening has been performed in both countries. To minimize the bias due to different methodology and interpretation, especially in the case of indirect immunofluorescence studies, autoantibody analysis was centralized in one laboratory from each country (La Paz University Hospital and University Hospital of Pisa). These determinations were performed in clinical routine laboratories with broad experience in autoimmunity and using validated methods. This study demonstrates a strong association of BSS with autoimmunity because $61.5 \%$ of the patients are positive for one or more autoantibodies, and $38.5 \%$ of them developed autoimmune-related disorders (Table 4). The patient BSS1 suffered from chronic HBV infection that could be associated with the presence of rheumatoid arthritis and markers of autoimmunity (RF and antidsDNA Ab). Several reports have illustrated the mechanisms involved in the loss of tolerance as a consequence of the immune response to HBV infection: such as molecular mimicry between HBV antigens and selfproteins, generation of immune complexes between HBV antigens and antibodies, promotion of apoptosis/ tissue damage. All the above mentioned mechanisms culminate in the exposure of intracellular antigens to the immune system and may end up with the development of a variety of autoimmune diseases [36, 37].

Since the first description of anti-adipocyte autoantibodies in a patient with AGL by Hübler and colleagues [38], its existence in BSS has been discussed. The results of the present study provide additional supportive evidence for a different autoimmune etiological basis for BSS according to which either local or systemic complement activation induced by autoantibodies against the C3 convertase of the AP or its individual components may play a relevant role.

We hypothesized that genetic and/or environmental factors a role in the disease onset. Among the putative genetic factors, HLA region on chromosome 6 is a reasonable candidate, as demonstrated through strong associations with a large variety of autoimmune or inflammatory diseases [39] such as type 1 diabetes and celiac disease [40, 41], Juvenile Autoimmune thyroiditis or ankylosing spondylitis with HLA-B*27 association [42, 43]. Although no HLA allele was shared by the majority of our patients, interestingly, the allele DRB1*11:03 were overrepresented in our cohort ( $31 \%$ carrier frequency vs $1.3 \%$ allelic frequency in the general population) [22]) (Table 3). There are hundreds of polymorphisms of HLA-DRB1 which have been associated with different autoimmune disorders as well as with immune response to infection and vaccines The association between HLA-DRB1*11 and autoimmune, infectious and cancer diseases has been previously reported for: systemic sclerosis, Henoch-Schönlein purpura, systemic juvenile idiopathic arthritis, Helicobacter pylori-positive idiopathic thrombocytopenic purpura, hairy cell leukaemia, cervical cancer, among others [44-49]. There are at least two reports dealing with an apparent association of the allele DRB1*11:03 with forms of juvenile idiopathic arthritis $[50,51]$. Future studies in larger patients' cohorts should address whether this allele is a real biomarker of BSS or not.

\section{Conclusions}

We here confirm that $\mathrm{C} 3$, and to a lower extent, C4 hypocomplementemia are common features of BSS, and that this pathology is frequently associated with autoimmunity. Moreover, besides $\mathrm{C} 3 \mathrm{NeF}$, other autoantibodies directed against components of the $\mathrm{C} 3$ convertase of the AP (anti-C3, anti-FB and anti-P) are present in a significant proportion of patients from our cohort. Finally, an association with the HLA allele DRB1*11:03 was observed, suggesting a potential role of this variant as a marker of the disease.

\section{Methods \\ Patients}

Thirteen patients were diagnosed with BSS on the basis of fat loss during childhood or adulthood affecting upper areas of the body, and having ruled out other causes of fat loss. The diagnosis was made based on standardized criteria [1]. The presence of other autoimmune diseases can be of support of the diagnosis. Laboratory findings as low serum $\mathrm{C} 3$ and the presence of $\mathrm{C} 3 \mathrm{NeF}$ were used during the diagnosis. Familial Partial Lipodystrophy (FPL) was also excluded based on the natural course of the disease, clinical features, age at onset, and absence of pathogenic variants in FPL-related genes (LMNA, PPARG, PLIN1, CIDEC, LIPE, ADRA2A, AKT2). No consanguinity was reported in any case. 


\section{Biological samples}

We collected serum samples from 13 patients with BSS (9 from Spain, and 4 from Italy) and 20 healthy subjects (NHS) (60\% female, aged 25-61 years). Controls and patients are not matched for age. Serum and EDTA plasma samples were obtained under standard conditions upon informed consent from the donors; blood was collected in plain tubes, allowed to clot at room temperature, and centrifuged for $10 \mathrm{~min}$ at $4{ }^{\circ} \mathrm{C}$. Serum and plasma were then aliquoted and stored frozen at $-80^{\circ} \mathrm{C}$ until their use.

\section{Measurement of the levels of complement system proteins}

Serum C3 and C4 levels were measured by nephelometry (Siemens Healthcare, Erlangen, Germany). FB and P levels were measured using in-house ELISA assays, previously described by our group [23].

\section{C3NeF detection by enzyme-linked immunosorbent assay (ELISA)}

$\mathrm{C} 3 \mathrm{NeF}$ detection in serum samples was performed as described previously by Paixão- Cavalcante and collaborators [24] with several modifications. Briefly, residual $\mathrm{Bb}$ was detected using a monoclonal anti-Bb antibody (A227, Quidel) $\left(1: 500 ; 1 \mathrm{~h}, 37^{\circ} \mathrm{C}\right)$, followed by peroxidase-conjugated goat anti-mouse IgG (Jackson Immunoresearch, West Grove, PA, USA) (1:5000; $1 \mathrm{~h})$. Color was developed using o-phenylenediamine dihydrochloride (Sigma-Aldrich, Madrid, Spain) and absorbance was measured at $492 \mathrm{~nm}$. Samples were considered positive when optical density was higher than 0.3 units of absorbance.

\section{Detection of autoantibodies against alternative pathway components ( $\mathrm{C} 3, \mathrm{FB}$ and $\mathrm{P})$ and regulators ( $\mathrm{FI}$ and $\mathrm{FH})$ by ELISA}

ELISA plates were coated with $100 \mathrm{ng} /$ well of purified C3, FB, FI, FH, or P. Plates were blocked with PBS-BSA $3 \%$ in case of $\mathrm{FB}$ and $\mathrm{P}$, and with PBS-BSA $0.1 \%$ in $\mathrm{FI}$ and C3. Serum samples were diluted in PBS-BSA 0.1\%, and binding of autoantibodies was detected with polyclonal anti-human IgG-HRP conjugated antibody (Jackson Immunoresearch) in ABTS substrate, as described by Nozal et al. [52]. Factor $\mathrm{H}$ autoantibodies were searched following methods previously described by Abarrategui-Garrido et al. [53]

\section{HLA genotyping}

HLA typing has been performed by two different laboratories. The methodology developed by each laboratory is described below:

- Spain: HLA-A, $-\mathrm{B},-\mathrm{C},-\mathrm{DRB1},-\mathrm{DQB1}$ and -DQA1 were studied by polymerase-chain reaction with sequence-specific oligonucleotide probes (PCR-SSOP) using commercial reagents (details available upon request). Those reagents distinguish all two-digit allelic groups and also discriminate most four-digit alleles commonly seen in the Spanish population [22, 40, 54-56]. In addition, we determined DPB1 phenotypes and confirmed DRB1*11:03 by sequence-based typing using local reagents. All HLA typing studies were performed following the quality standards issued by the European Federation for Immunogenetics.

- Italy: A single PCR reaction was used for each HLA locus. Luminex-based technology was applied to discriminate among the different human HLA alleles, by using sequence-specific oligonucleotide probes bound to color-coded microbeads in order to identify HLA alleles encoded by the DNA sample [57]. A flow analyzer identifies the fluorescent intensity SAPE on each microsphere. A software was used to assign positive or negative reactions based on the strength of the fluorescent signal.

\section{Screening of autoantibodies}

The screening of autoantibodies was performed by two different laboratories. The methodology developed by each laboratory is described below:

- Spain: Anti-cellular autoantibodies were tested by indirect immunofluorescence (IIF) on Hep-2 cells (Euroimmun, Luebeck, Germany), and ENA analysis was performed, either of IIF was positive or not, by Bioplex ANA Screen kit (Bio-Rad, Hercules, CA), which includes dsDNA, chromatin, centromere B, Scl70, RNP-68 kDa, RNP-A, Ro/SSA $52 \mathrm{kDa}, \mathrm{Ro} / \mathrm{SSA} 60 \mathrm{kDA}, \mathrm{La} / \mathrm{SSB}, \mathrm{Sm}$, $\mathrm{Sm} / \mathrm{RNP}$, Jo-1, and $\mathrm{P}$ ribosomal proteins as antigens. Rheumatoid factor (RF) was determined by nephelometry (Siemens Healthcare, Erlangen, Germany).

Anti-thyroid peroxidase (TPO-Ab) and antithyroglobulin autoantibodies (TG-Ab) were quantified by fluorescence enzyme immunoassay (EliA, PhadiaThermo Fisher, Freiburg, Germany). Anti-islet cells antibodies and anti-suprarrenal cortex autoantibodies detection was performed by IIF on monkey pancreas and monkey suprarenal gland tissues (Immco Diagnostics, Buffalo, NY and Biosystems, Barcelona, Spain, respectively). Anti-parietal cells autoantibodies (APCA), antimitochondrial and anti-smooth muscle autoantibodies were tested by IIF on rat liver, kidney and stomach substrates (Euroimmun, Luebeck, Germany).

- Italy: TPO-Ab and TG-Ab (AIA, Tosoh Bioscience Griesheim, Germany) and Anti-transglutaminase autoantibodies (Thermo Fisher Scientific, Waltham, USA) were detected by fluorescence enzyme immunoassay. Glutamic Acid Decarboxylase Autoantibodies (GAD-Ab) were tested by radioimmunoassay (Ria Medipan, Berlin Germany). APCA (Elisa Orgentc Diagnostika, Mainz, Germany), and 21-Hydroxylase (21-OH) Autoantibodies 
(Elisa RSR Cardiff, U.K) were quantified using commercial ELISA kits. ANA and dsDNA were tested by IIF (Euroimmun, Luebeck, Germany). Finally, Direct Antiglobulin Test (DAT) was analyzed by Column Agglutination (Ortho Clinical Diagnostics Pencoed, UK).

\section{Statistical analysis}

Statistical calculations were performed with Prism version 6.01 (GraphPad Software, La Jolla, CA, USA). Man-Whitney was used for comparisons among the groups. A $P$-value of $<0.05$ was considered statistically significant in all analyses.

\section{Supplementary information}

Supplementary information accompanies this paper at https://doi.org/10 1186/s13023-019-1292-1.

\section{Additional file 1: Figure S1. The alternative pathway of the} complement system and autoantibodies against its proteins. In the alternative pathway (AP), continuous, low-level activation of C3 by spontaneous hydrolysis of the internal C3 thioester, or C3 cleavage by plasma proteases, generates $\mathrm{C}_{3}\left(\mathrm{H}_{2} \mathrm{O}\right)$ or $\mathrm{C} 3 \mathrm{~b}$. Activation by the AP leads to the generation of $\mathrm{C} 3$ convertase complexes $(\mathrm{C} 3 \mathrm{bBb})$ that cleave $\mathrm{C} 3$ into $\mathrm{C} 3 \mathrm{a}$ and $\mathrm{C} 3 \mathrm{~b}$. Additionally, the AP C3 convertase can bind properdin (P), a positive regulator that stabilizes the enzyme, extending its half-life more than 10-fold. The activation of the AP is controlled by two different soluble regulatory proteins, as factor $\mathrm{H}(\mathrm{FH})$ and factor I ( $\mathrm{Fl})$. C3 nephritic factor $(\mathrm{C} 3 \mathrm{NeF})$ is one of the most known autoantibodies which recognized a neo-epitope in the $\mathrm{C} 3$ convertase. Other autoantibodies against complement components ( $\mathrm{C} 3, \mathrm{FB}$ or $\mathrm{P})$ and regulators ( $\mathrm{FH}$ or $\mathrm{FI})$, some of them with functional activities, have been shown in the figure. Figure S2. Lack of correlation between complement levels and age. In 20 healthy subjects no correlations were found among age and the levels of (A) C3, (B) C4 (C) factor B (FB) and (D) properdin (P). These data were obtained using the Spearman Rank correlation coefficient. $P<0.05$ were considered statistically significant. Figure S3. Gender does not significantly modify complement levels in our cohort of healthy subjects. In 20 healthy subjects complement levels of (A) C3, (B) C4, (C) factor B (FB) and $(D)$ properdin $(P)$ were measured. The mean of concentration in males and females was analyzed by the Mann Whitney test. $P<0.05$ was considered statistically significant.

\section{Abbreviations}

AGL: Acquired generalized lipodystrophy; AP: Alternative pathway; APCA: Anti-parietal cells autoantibodies; BSS: Barraquer-Simons syndrome; C3G: C3 glomerulopathy; C3GN: C3 glomerulonephritis; C3NeF: C3 nephritic factor; DAT: Direct Antiglobulin test; DDD: Dense deposit disease; FB: Factor B; FD: Factor D; FH: Factor H; Fl: Factor I; FPL: Familial Partial Lipodystrophy; GAD-Ab: Glutamic Acid Decarboxylase Autoantibodies; HBV: Hepatitis B Virus; HLA: Human Leukocyte antigen; P: Properdin; RF: Rheumatoid factor; TGAb: Anti-thyroglobulin autoantibodies; TPO-Ab: Anti-thyroid peroxidase

\section{Acknowledgements}

We would like to thank our patients, their families and clinicians for being part of this study.

\section{Authors' contributions}

FC designed the study and wrote the article. FC, ML-T, DA-V, FS, SM, CP, and $G C$ recruited the patients and selected clinical, demographical and laboratory data. FC, SGar, PN determined the complement components levels. MM, CV, SF and SGab performed HLA genotyping. FC, SGar, PN, SM and CP performed the screening of autoantibodies. All authors reviewed and approved the final version of the article.

\section{Funding}

This work was supported by Instituto de Salud Carlos III (Ministerio de Ciencia, Innovación y Universidades, Gobierno de España) and Fondos FEDER
(PI15-00255 to M.L-T. and PI08-1449 to D.A-V.), Complemento II-CM network (B2017/BMD3673 to M.L-T), the intramural research program of the Xunta de Galicia (Programa de Consolidación e Estructuración de Unidades de Investigación Competitivas, grant ED341b 2017/19 to D.A-V.), the Asociación Española de Familiares y Afectados de Lipodistrofias (AELIP) (to D.A-V., to F.C. and to P.N.).

\section{Availability of data and materials}

The data that support the findings of this study are available from the corresponding author upon reasonable request.

\section{Ethics approval and consent to participate}

All procedures performed in studies involving human participants were in accordance with the ethical standards of the Clinical Research Ethics Committee of La Paz University Hospital (project code PI-2276) and with the 1964 Helsinki declaration and its later amendments or comparable ethical standards. All participants provided written informed consent for participation in the study and for the publication of their clinical and biochemical information.

\section{Consent for publication}

Not applicable.

\section{Competing interests}

The authors declare that they have no competing interests.

\section{Author details}

${ }^{1}$ Complement Research Group, Hospital La Paz Institute for Health Research (IdiPAZ), La Paz University Hospital, Paseo de la Castellana, 261, 28046 Madrid, Spain. ${ }^{2}$ Center for Biomedical Network Research on Rare Diseases (CIBERER U754), Madrid, Spain. ${ }^{3}$ Obesity and Lipodystrophy Centre at the Endocrinology Unit, University Hospital of Pisa, Pisa, Italy. ${ }^{4}$ Unit of Immunology, La Paz University Hospital, Madrid, Spain. ${ }^{5}$ Immunogenetics and Histocompatibility, Instituto de Investigación Sanitaria Puerta de Hierro, Madrid, Spain. ${ }^{6}$ Immunogenetics laboratory, University Hospital of Pisa, Pisa, Italy. ${ }^{7}$ Thyroid and Metabolic Diseases Unit (U.E.T.eM.), Centro Singular de Investigación en Medicina Molecular e Enfermidades Crónicas (CIMUS-IDIS), School of Medicine, Universidad de Santiago de Compostela, Santiago de Compostela, Spain. ${ }^{8}$ Universidad Autónoma de Madrid, Madrid, Spain.

Received: 12 September 2019 Accepted: 29 December 2019

Published online: 10 January 2020

\section{References}

1. Araújo-Vilar D, Santini F. Diagnosis and treatment of lipodystrophy: a stepby-step approach. J Endocrinol Investig. 2019;42:61-73.

2. Garg A. Acquired and inherited lipodystrophies. N Engl J Med. 2004;350: 1220-34.

3. Hussain I, Garg A. Lipodystrophy syndromes. Endocrinol Metab Clin N Am. 2016;45:783-97

4. Brown RJ, Araujo-Vilar D, Cheung PT, Dunger D, Garg A, Jack M, et al. The diagnosis and Management of Lipodystrophy Syndromes: a multi-society practice guideline. J Clin Endocrinol Metab. 2016;101:4500-11.

5. Misra A, Peethambaram A, Garg A. Clinical features and metabolic and autoimmune derangements in acquired partial lipodystrophy: report of 35 cases and review of the literature. Medicine (Baltimore). 2004;83:18-34.

6. Ozgen Saydam B, Sonmez M, Simsir IY, Erturk MS, Kulaksizoglu M, Arkan T, et al. A subset of patients with acquired partial lipodystrophy developing severe metabolic abnormalities. Endocr Res. 2019;44:46-54.

7. Pope E, Janson A, Khambalia A, Feldman B. Childhood acquired lipodystrophy: a retrospective study. J Am Acad Dermatol. 2006;55:947-50.

8. Sissons JG, West RJ, Fallows J, Williams DG, Boucher BJ, Amos N, et al. The complement abnormalities of lipodystrophy. N Engl J Med. 1976;294:461-5.

9. López-Lera A, Corvillo F, Nozal P, Regueiro JR, Sánchez-Corral P, LópezTrascasa M. Complement as a diagnostic tool in immunopathology. Semin Cell Dev Biol. 2019;85:86-97.

10. Levy Erez D, Meyers KE, Sullivan KE. C3 nephritic factors: a changing landscape. J Allergy Clin Immunol. 2017;140:57-9.

11. Sethi S, Fervenza FC. Standardized classification and reporting of glomerulonephritis. Nephrol Dial Transplant. 2019;34:193-9. 
12. Tan $\mathrm{Y}$, Zhao M-H. Complement in glomerular diseases: complement in glomerular diseases. Nephrology. 2018;23:11-5.

13. Smith RJH, Appel GB, Blom AM, Cook HT, D'Agati VD, Fakhouri F, et al. C3 glomerulopathy - understanding a rare complement-driven renal disease. Nat Rev Nephrol. 2019;15:129-43.

14. Rosen BS, Cook KS, Yaglom J, Groves DL, Volanakis JE, Damm D, et al. Adipsin and complement factor D activity: an immune-related defect in obesity. Science. 1989;244:1483-7.

15. White RT, Damm D, Hancock N, Rosen BS, Lowell BB, Usher P, et al. Human adipsin is identical to complement factor $D$ and is expressed at high levels in adipose tissue. J Biol Chem. 1992;267:9210-3.

16. Wu X, Hutson I, Akk AM, Mascharak S, Pham CTN, Hourcade DE, et al. Contribution of adipose-derived factor D/Adipsin to complement alternative pathway activation: lessons from Lipodystrophy. J Immunol. 2018;200:2786-97.

17. Pattrick M, Luckett J, Yue L, Stover C. Dual role of complement in adipose tissue. Mol Immunol. 2009;46:755-60.

18. Moreno-Navarrete JM, Martínez-Barricarte R, Catalán V, Sabater M, GómezAmbrosi J, Ortega FJ, et al. Complement factor $\mathrm{H}$ is expressed in adipose tissue in association with insulin resistance. Diabetes. 2010;59:200-9.

19. Gauvreau D, Roy C, Tom F-Q, Lu H, Miegueu P, Richard D, et al. A new effector of lipid metabolism: complement factor properdin. Mol Immunol. 2012:51:73-81.

20. Matsunaga H, Iwashita M, Shinjo T, Yamashita A, Tsuruta M, Nagasaka S, et al. Adipose tissue complement factor B promotes adipocyte maturation. Biochem Biophys Res Commun. 2018;495:740-8.

21. Mathieson PW, Würzner R, Oliveria DB, Lachmann PJ, Peters DK. Complementmediated adipocyte lysis by nephritic factor sera. J Exp Med. 1993;177:1827-31.

22. Montero-Martín G, Mallempati KC, Gangavarapu S, Sánchez-Gordo F, HerreroMata MJ, Balas A, et al. High-resolution characterization of allelic and haplotypic HLA frequency distribution in a Spanish population using highthroughput next-generation sequencing. Hum Immunol. 2019;80:429-36.

23. Convillo F, Bravo García-Morato M, Nozal P, Garrido S, Tortajada A, Rodríguez de Córdoba S, et al. Serum properdin consumption as a biomarker of C5 convertase dysregulation in C3 glomerulopathy. Clin Exp Immunol. 2016;184:118-25.

24. Paixão-Cavalcante D, López-Trascasa M, Skattum L, Giclas PC, Goodship TH, de Córdoba SR, et al. Sensitive and specific assays for C3 nephritic factors clarify mechanisms underlying complement dysregulation. Kidney Int. 2012;82:1084-92.

25. Ohi $\mathrm{H}$, Yasugi T. Occurrence of $\mathrm{C} 3$ nephritic factor and C4 nephritic factor in membranoproliferative glomerulonephritis (MPGN). Clin Exp Immunol. 1994; 95:316-21.

26. Savage DB, Semple RK, Clatworthy MR, Lyons PA, Morgan BP, Cochran EK, et al. Complement abnormalities in acquired lipodystrophy revisited. J Clin Endocrinol Metab. 2009;94:10-6.

27. Eren E, Özkan TB, Çakır EDP, Sağlam H, Tarım Ö. Acquired generalized lipodystrophy associated with autoimmune hepatitis and low serum C4 level. J Clin Res Pediatr Endocrinol. 2010;2:39-42.

28. Gaya da Costa M, Poppelaars F, van Kooten C, Mollnes TE, Tedesco F, Würzner R, et al. Age and Sex-Associated Changes of Complement Activity and Complement Levels in a Healthy Caucasian Population. Front Immunol. 2018; [cited 2019 Nov 15];9. Available from: https://www.ncbi.n/m.nih.gov/ pmc/articles/PMC6255829/.

29. Norman ME, Gall EP, Taylor A, Laster L, Nilsson UR. Serum complement profiles in infants and children. J Pediatr. 1975;87:912-6.

30. Strobel S, Zimmering M, Papp K, Prechl J, Józsi M. Anti-factor B autoantibody in dense deposit disease. Mol Immunol. 2010;47:1476-83.

31. Chen Q, Müller D, Rudolph B, Hartmann A, Kuwertz-Bröking E, Wu K, et al. Combined C3b and factor B autoantibodies and MPGN type II. N Engl J Med. 2011;365:2340-2

32. Marinozzi MC, Roumenina LT, Chauvet S, Hertig A, Bertrand D, Olagne J, et al. Anti-factor B and anti-C3b autoantibodies in C3 Glomerulopathy and lgassociated Membranoproliferative GN. J Am Soc Nephrol. 2017;28:1603-13.

33. Vasilev W, Noe R, Dragon-Durey M-A, Chauvet S, Lazarov VJ, Deliyska $B P$, et al. Functional characterization of autoantibodies against complement component C3 in patients with lupus nephritis. J Biol Chem. 2015;290:25343-55

34. Vasilev W, Radanova M, Lazarov VJ, Dragon-Durey M-A, Fremeaux-Bacchi V, Roumenina LT. Autoantibodies against C3b-functional consequences and disease relevance. Front Immunol. 2019;10:64.

35. Muto Y, Fujimura T, Kakizaki A, Tsuchiyama K, Kusakari Y, Aiba S. Adult-onset acquired partial Lipodystrophy accompanied by rheumatoid arthritis. Case Rep Dermatol. 2015;7:70-4.
36. Maya R, Gershwin ME, Shoenfeld Y. Hepatitis B virus (HBV) and autoimmune disease. Clin Rev Allergy Immunol. 2008;34:85-102.

37. Ram M, Anaya J-M, Barzilai O, Izhaky D, Porat Katz B-S, Blank M, et al. The putative protective role of hepatitis $B$ virus (HBV) infection from autoimmune disorders. Autoimmun Rev. 2008;7:621-5.

38. Hübler A, Abendroth K, Keiner T, Stöcker W, Kauf E, Hein G, et al. Dysregulation of insulin-like growth factors in a case of generalized acquired lipoatrophic diabetes mellitus (Lawrence syndrome) connected with autoantibodies against adipocyte membranes. Exp Clin Endocrinol Diabetes. 1998;106:79-84.

39. McDevitt HO, Bodmer WF. HL-A, immune-response genes, and disease. Lancet. 1974;1:1269-75.

40. Balas A, García-Sánchez F, Vicario JL. A new DQA1 allele (DQA1*0510) in a Spanish celiac disease patient. Tissue Antigens. 2010;75:287-8.

41. Farina F, Picascia S, Pisapia L, Barba P, Vitale S, Franzese A, et al. HLA-DQA1 and HLA-DQB1 alleles, conferring susceptibility to celiac disease and type 1 diabetes, are more expressed than non-predisposing alleles and are coordinately regulated. Cells. 2019;8:751.

42. REGISPONSER group, Arévalo M, Gratacós Masmitjà J, Moreno M, Calvet J, Orellana C, et al. Influence of HLA-B27 on the Ankylosing Spondylitis phenotype: results from the REGISPONSER database. Arthritis Res Ther. 2018;20:221.

43. Kanga U, Tandon N, Marwaha RK, Khanna R, Bhattacharya B, Singh S, et al. Immunogenetic association and thyroid autoantibodies in juvenile autoimmune thyroiditis in North India. Clin Endocrinol. 2006; [cited 2019 Aug 8]. Available from: https://onlinelibrary.wiley.com/doi/abs/10.1111/ j.1365-2265.2006.02511.x.

44. Arons E, Adams S, Venzon DJ, Pastan I, Kreitman RJ. Class II human leucocyte antigen DRB1*11 in hairy cell leukaemia patients with and without haemolytic uraemic syndrome. Br J Haematol. 2014;166:729-38.

45. Gladman DD, Kung TN, Siannis F, Pellett F, Farewell VT, Lee P. HLA markers for susceptibility and expression in scleroderma. J Rheumatol. 2005;32:1481-7.

46. He X, Yu C, Zhao P, Ding Y, Liang X, Zhao Y, et al. The genetics of HenochSchönlein purpura: a systematic review and meta-analysis. Rheumatol Int. 2013:33:1387-95.

47. Veneri D, De Matteis G, Solero P, Federici F, Zanuso C, Guizzardi E, et al. Analysis of B- and T-cell clonality and HLA class II alleles in patients with idiopathic thrombocytopenic purpura: correlation with helicobacter pylori infection and response to eradication treatment. Platelets. 2005;16:307-11.

48. Ombrello MJ, Remmers EF, Tachmazidou I, Grom A, Foell D, Haas J-P, et al. HLA-DRB1*11 and variants of the MHC class II locus are strong risk factors for systemic juvenile idiopathic arthritis. Proc Natl Acad Sci U S A. 2015;112:15970-5.

49. Theodorou I, Abel L, Mauro F, Duprey B, Magnac C, Payelle-Brogard B, et al. High occurence of DRB1 11 in chronic lymphocytic leukaemia families. Br J Haematol. 2002;119:713-5.

50. Haas JP, Truckenbrodt H, Paul C, Hoza J, Scholz S, Albert ED. Subtypes of HLA-DRB $1{ }^{*} 03,{ }^{*} 08,{ }^{*} 11,{ }^{*} 12,{ }^{*} 13$ and ${ }^{*} 14$ in early onset pauciarticular juvenile chronic arthritis (EOPA) with and without iridocyclitis. Clin Exp Rheumatol. 1994;12(Suppl 10):S7-14.

51. Hollenbach JA, Thompson SD, Bugawan TL, Ryan M, Sudman M, Marion M, et al. Juvenile idiopathic arthritis and HLA class I and class II interactions and age-at-onset effects. Arthritis Rheum. 2010;62:1781-91.

52. Nozal P, Garrido S, Martínez-Ara J, Picazo ML, Yébenes L, ÁlvarezDoforno $\mathrm{R}$, et al. Case report: lupus nephritis with autoantibodies to complement alternative pathway proteins and C3 gene mutation. BMC Nephrol. 2015;16:40

53. Abarrategui-Garrido C, Martínez-Barricarte R, López-Trascasa M, de Córdoba SR, Sánchez-Corral P. Characterization of complement factor H-related (CFHR) proteins in plasma reveals novel genetic variations of CFHR1 associated with atypical hemolytic uremic syndrome. Blood. 2009;1 14:4261-71.

54. Balas A, García-Sánchez F, Vicario LJ. The new HLA-CW*0442 allele possibly generated by a recombination event involving $C W^{*} 04010101$ and $C W^{*} 1801$. Tissue Antigens. 2010;75:82-3.

55. Balas A, García-Sánchez F, Vicario JL. HLA-B*4907 and B*490101 differs by residue 163 placed in a dominant serologic epitope. Tissue Antigens. 2010;75:286-7.

56. Balas A, García-Sánchez F, Vicario JL. Sequencing of a new HLA-DRB1*16 allele (DRB1*1615) with valine at residue 86. Tissue Antigens. 2010;75:288-9.

57. Testi M, Andreani M. Luminex-based methods in high-resolution HLA typing. Methods Mol Biol. 2015;1310:231-45. 


\section{Publisher's Note}

Springer Nature remains neutral with regard to jurisdictional claims in published maps and institutional affiliations.

- fast, convenient online submission

- thorough peer review by experienced researchers in your field

- rapid publication on acceptance

- support for research data, including large and complex data types

- gold Open Access which fosters wider collaboration and increased citations

- maximum visibility for your research: over $100 \mathrm{M}$ website views per year

At $\mathrm{BMC}$, research is always in progress. 